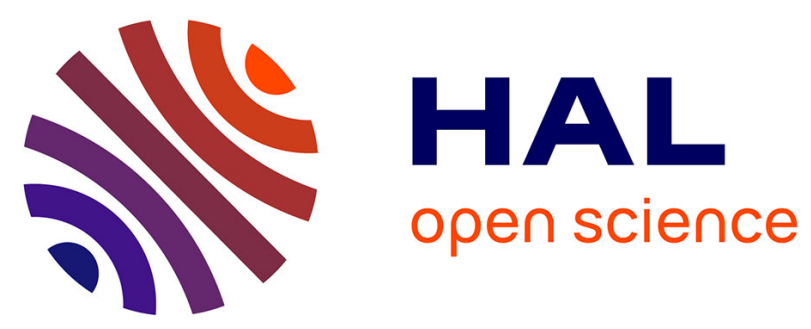

\title{
Time trends of syphilis and HSV-2 co-infection among men who have sex with men in the German HIV-1 Seroconverter Cohort from 1996 through 2007
}

Nadine Spielmann, Dieter Muenstermann, Hans-Jochen Hagedorn, Matthias an Der Heiden, Claudia Houareau, Barbara Gunsenheimer-Bartmeier, Claudia Kuecherer, Katrin Keeren, Osamah Hamouda, Ulrich Marcus

\section{To cite this version:}

Nadine Spielmann, Dieter Muenstermann, Hans-Jochen Hagedorn, Matthias an Der Heiden, Claudia Houareau, et al.. Time trends of syphilis and HSV-2 co-infection among men who have sex with men in the German HIV-1 Seroconverter Cohort from 1996 through 2007. Sexually Transmitted Infections, 2010, 86 (5), pp.331. 10.1136/sti.2009.040857 . hal-00570213

\section{HAL Id: hal-00570213 \\ https://hal.science/hal-00570213}

Submitted on 28 Feb 2011

HAL is a multi-disciplinary open access archive for the deposit and dissemination of scientific research documents, whether they are published or not. The documents may come from teaching and research institutions in France or abroad, or from public or private research centers.
L'archive ouverte pluridisciplinaire $\mathbf{H A L}$, est destinée au dépôt et à la diffusion de documents scientifiques de niveau recherche, publiés ou non, émanant des établissements d'enseignement et de recherche français ou étrangers, des laboratoires publics ou privés. 
Time trends of syphilis and HSV-2 co-infection among men who have sex with men in the German HIV-1 Seroconverter Cohort from 1996 through 2007

Nadine Spielmann ${ }^{1}$, Dieter Münstermann ${ }^{2}$, Hans-Jochen Hagedorn ${ }^{2}$, Matthias an der Heiden ${ }^{1}$, Claudia Houareau ${ }^{1}$, Barbara Gunsenheimer-Bartmeyer ${ }^{1}$, Claudia Kücherer ${ }^{3}$, Katrin Keeren ${ }^{3}$, Osamah Hamouda ${ }^{1}$, Ulrich Marcus ${ }^{1}$ and the German HIV-1 Seroconverter Study Group

1 Department of Infectious Disease Epidemiology, HIV/AIDS, STI Unit, Robert Koch Institute, Berlin, Germany

${ }^{2}$ Labor Krone, Bad Salzuflen, Germany

3 Project HIV Variability and Molecular Epidemiology, Robert Koch Institute, Berlin, Germany

Contact: MarcusU@ @ki.de, Robert Koch Institute, DGZ-Ring 1, 13086 Berlin. 


\section{Abstract (248 words)}

\section{Objectives}

Numbers of newly diagnosed HIV infections among men who have sex with men (MSM) in Germany increased after the year 2000. We sought to explore trends in STI co-infections around the time of HIV seroconversion in patients from the German HIV-1 seroconverter cohort in the years 1996 through 2007.

\section{Methods}

MSM from the cohort were included for secondary analysis, if seroconversion occurred between 1996 and 2007 and if a blood sample taken within two years after HIV infection was available for further testing. Samples were tested for antibodies against Treponema pallidum and HSV-2. A classification system was developed to assign the chronology of syphilis and HIV-1 infection.

\section{Results}

Data of 1,052 MSM were eligible for analysis. Overall seroprevalence of syphilis markers was $26 \%$, increasing from $10 \%$ (1996-1999) to $35 \%$ (2005). Among HIV seroconverters with positive syphilis antibodies, $32 \%(\mathrm{n}=88)$ were rated as having had coincident infections with HIV and syphilis. Coincident syphilis infection at HIV diagnosis increased substantially ( $\mathrm{p}<0.001$ ) from $2.3 \%$ in 2000 to $16.9 \%$ in 2003 ; and thereafter declined to $4.3 \%$ in 2007 . Mean HSV-2 antibody prevalence was $40.5 \%$, mean anti-HSV-2 IgM prevalence was $11.2 \%$, with no significant change over time.

\section{Discussion}

We found a stable prevalence of HSV-2 infection and increasing prevalence of syphilis infection around the time of HIV acquisition among MSM in Germany. Time course and rate 
of co-infections suggest that a re-emerging syphilis co-epidemic among MSM after 2000 could have contributed to an increase of HIV incidence by enhancing HIV transmission probability.

\section{Keywords:}

Syphilis, HSV-2, HIV transmission, STI co-factor, Men who have sex with men (MSM) 


\section{Objectives}

Epidemiological data show an increase in newly diagnosed HIV infections among men who have sex with men (MSM) - in Germany as well as other countries in Western Europe, North America, and Australia after the year 2000 [1]. Simultaneously, increasing syphilis prevalence and incidence have been identified in the same subpopulation group [2]. In Germany, the number of newly reported syphilis cases more than doubled since 2001 , and reached mean values of approximately 4/1,000 MSM in 2004 and the following years [3; 4].

It has been hypothesized that a rise in infections with HIV can be caused by an increase of the per-contact probability for HIV transmission due to ulcerative STIs like syphilis and anogenital herpes [5]. In this report, we sought to explore trends in coincident HIV, syphilis and HSV-2 infection in HIV patients from the German HIV-1 seroconverter cohort in the years 1996 through 2007.

\section{Methods}

The German HIV-1 Seroconverter Study is a national prospective multi-centre observational cohort that started in 1997 . The study was approved by the ethical committee of the Charite University Clinic in Berlin. The study design has been described in detail elsewhere [6]. In brief, as of December 31, 2007, a total of 1,285 blood samples of HIV-1 seroconverters were available for screening for syphilis, HSV-1 and HSV-2 co-infection. The first blood sample taken after enrolment was used for analysis if the blood sample was taken within two years after the estimated date of HIV seroconversion. The serologic tests for syphilis, HSV-1 and HSV-2 were performed with frozen plasma samples, including ARCHITECT $^{\circledR}$ Syphilis TP chemiluminescence microparticle immuno assay for screening and Fluorescent Treponemal Antibody Absorption (FTA-ABS) test for confirmation, and the T. pallidum Particle Agglutination (TPPA) Assay for the determination of treponemal antibody titers in reactive samples. The presence of HSV-1, HSV-2 IgG and HSV-2 IgM antibodies in the samples were 
tested using ELISA test kits for the quantitative determination of type specific antibodies against the glycoproteins G-1 (HSV-1) and G-2 (HSV-2).

For the assessment of syphilis disease activity in reactive samples two additional tests were performed: As a lipoidal antibody test a modification of the VDRL test, the RPR reditest ${ }^{\circledR}$ was used. Treponemal IgM antibodies were determined quantitatively by the IgM-FTA-ABS test after elimination of the $\operatorname{IgG}$ fraction from the sample with an anti-IgG serum, using the same antigen and sorbent as for the IgG-FTA-ABS test and a rabbit anti-human IgM-FITC conjugate. IgM antibody titers less than 1:10 were classified as negative, titers of 1:10 as borderline, titers of 1:20 as weak positive and titers 1:40 or more as positive. Serological testing was performed at Labor Krone, Bad Salzuflen, Germany.

Further information on the study subjects were provided by their respective HIV health care provider, including information on gender and the supposed route of HIV transmission (injecting drug user, migrant from a country with high HIV prevalence, heterosexual, MSM, unknown). For the calculation of a chronological relation between HIV seroconversion and syphilis infection, analysis was restricted to male subjects reported to be MSM or with unknown route of HIV transmission (MSM/u).

Based on (i) antibody patterns for syphilis, (ii) the time interval between blood sampling and estimated date of HIV infection, and (iii) information about a history of syphilis within the previous two years (yes/ no), provided by the study subjects' HIV health care facility, HIV seroconverters were assigned to the following three groups: (1) active syphilis predated HIV seroconversion, (2) active syphilis coincident with HIV seroconversion, and (3) syphilis acquired after HIV seroconversion. If a history of syphilis was reported, we assumed that adequate antibiotic treatment had been provided. 
- If the blood sample was taken within three months after the estimated time point of HIV infection, groups were defined as follows:

Group 1: $\quad$ IgM titer $<1: 40$ and VDRL titer $<1: 8$

Group 2: $\quad \operatorname{IgM}$ titer $\geq 1: 40$ or VDRL titer $\geq 1: 8$

Group 3: not applicable.

- If the blood sample was taken between four to twenty four months after the estimated time of HIV infection, groups were defined as follows:

Group 1: $\quad \operatorname{IgM}$ titer $<1: 10$ and VDRL titer $<1: 2$

Group 2: $\quad$ IgM titer $>1: 10$ or VDRL titer $>1: 2$ and not meeting definition for group 3

Group 3: $\quad$ IgM titer $\geq 1: 80$ if blood sample was taken between 12-24 months after infection

IgM titer $\geq 1: 320$ if sample was taken within 7-12 months after infection IgM titer $\geq 1: 1280$ if sample was taken within 4-6 months after infection.

- Seroconverters with IgM titer =1:10 or VDRL titer =1:2 were individually grouped based on information about history of syphilis in the previous 2 years.

Group 1: No history of syphilis within the previous two years

Group 2: History of syphilis within the previous two years

To evaluate potential confounders for the observed trends on HIV-syphilis co-infection, we analysed time trends in the study sample of HIV-1 seroconverters for the following parameters:

- As surrogate markers for 'recruitment bias': age at HIV infection, and proportion of HIV seroconverters recruited in Berlin (largest city in Germany); 
- as markers of a 'group assignation bias': ratio of documented (last negative/ first positive HIV test result) vs. acute (according to laboratory criteria: i) detectable HIV-1 RNA or p24 antigen combined with a negative or indeterminate ELISA result or ii) reactive HIV-1-ELISA combined with a negative or indeterminate immunoblot-result followed by confirmation of complete seroconversion within 6 months) HIV seroconversion; mean time between last negative and first positive HIV test result; mean time between calculated date of infection and first available blood sample;

- as an indicator for changes in sexual risk behaviour: proportion of seroconverters with HSV-1 and HSV-2 antibodies and with HSV-2 IgM antibodies (it has been shown that incident infection with HSV-2 in MSM is significantly associated with a variety of anal sex practices with casual partners [7]).

We used a log-linear regression model (Poisson regression [8]) to detect trends in the syphilis co-infection rate in the group of MSM/u seroconverters. We investigated coincident and preceding episodes of syphilis separately and checked whether other variables had a significant influence. Statistical significance was defined as a $P$-value of $<0.05$. STATA version 10.1 was used for statistics.

\section{Results}

Of 1,285 HIV-1 seroconverters included in the cohort, 1,105 were reported as MSM/u, among these 1,052 had a blood sample taken within two years after the estimated date of HIV infection. $276 \mathrm{MSM} / \mathrm{u}$ (26.2\%) were positive for treponemal antibodies (TPPA), the median distance to the estimated time point of HIV infection was 5 months. A single subject could not be assigned to one of the three groups as described above. Out of the remaining $275 \mathrm{MSM} / \mathrm{u}$ (MSM =270; unknown risk =5), 88 (31.9\%) were assigned to the group with coinciding infections with HIV and syphilis (group 2), 176 men (63.8\%) to the group with treponemal 
infection preceding HIV infection (group 1), and 11 men to the group where HIV infection was followed by syphilis infection (group 3; see Figure 1).

The overall proportion of syphilis seromarkers among MSM/u HIV seroconverters significantly $(\mathrm{p}<0.001)$ increased from $9.8 \%(1996-1999)$ to $34.7 \%(2005)$, and thereafter declined to $23.4 \%$ (2007). The proportion of men with syphilis infection preceding HIV infection (group 1) increased from 6.1\% (1996-1999) to $22.7 \%$ (2004) (p<0.001), and thereafter slightly declined to $19.1 \%$ (2007).

Coincident syphilis infection at HIV seroconversion (group 2) increased from 2.3\% (1996$1999)$ to $16.9 \%(2003)(\mathrm{p}<0.001)$ and thereafter declined to $4.3 \%$ (2007) (see Table 1).

The mean age at HIV infection increased from 32 to 35 years over the observation period. The proportion of seroconverters recruited in Berlin, the city with the largest gay population in Germany, remained stable around $70 \%$. The proportion of documented to acute HIV seroconversion and the mean time interval between HIV infection and blood sample declined stepwise over time. In all seroconverters, time between last negative and first positive test result did not change significantly during the observation period. The proportion of seroconverters with antibodies against HSV-1 fluctuated around 90\%, the proportion with HSV-2 antibodies around $40 \%$, with no clear trends over time. The same was observed regarding the proportion with HSV-2 IgM antibodies (prevalence between 7 and $16 \%$ - see Table 1).

Analyzing time trends by Poisson regression, we found a significant increase of coincident syphilis infections in MSM/u-seroconverters in the time period from 1996 up to 2003: on average the incidence rate increased by $51.8 \%$ (95\% C.I. $20.6 \%-91.1 \%$ ) per year. Other variables showed no significant influence on the incidence of coincident syphilis. 
For syphilis preceding HIV infection we found a significant increase between 1996 and 2004. Moreover, in a multivariate model we found in the same period an additional influence of age at seroconversion and place of residence. On average the syphilis antibody prevalence rate increased by $19.0 \%$ (95\% C.I. $6.6 \%-32.8 \%$ ) per year; for each year of age the prevalence of a preceding syphilis infection increased by $2.9 \%$ (95\% C.I. $0.4 \%-5.5 \%)$; seroconverters from Berlin had a $84.9 \%$ (95\% C.I. $2.1 \%$ - 234.8\%) higher prevalence compared to other seroconverters (see Table 2).

\section{Discussion}

We found a significant increase in the prevalence of syphilis antibodies in MSM HIVseroconverters between 2000 and 2005. During this period the number of reported syphilis cases in Germany more than doubled. A more detailed analysis of syphilis antibody patterns suggested that a large proportion $(\sim 2 / 3)$ of these syphilis antibody positive HIV seroconverters may have acquired syphilis before HIV, but also the proportion of probably coincident HIV and syphilis infections increased significantly from before 2000 to 2003 with leveling off and decline thereafter. Interestingly, in contrast to increasing prevalence of syphilis antibodies in HIV seroconverters, we did not find any consistent changes for HSV-2 antibody prevalence. The presence of HSV-1 antibody was almost universal ( 90\%). Prevalence of HSV-1 and HSV-2 antibodies was comparable to reports about HIV-positive MSM from the Netherlands and the United Kingdom [9; 10].

We were able to analyze time trends for only a limited set of other parameters in the seroconverter cohort. We observed a slightly increasing trend for age at HIV seroconversion (reflected as well in the national surveillance data for HIV and syphilis in MSM), a trend for improved recognition of acute seroconversion (declining ratio of documented/ acute seroconversion), and faster recruitment of seroconverters into the cohort (declining time delay 
between seroconversion and blood sample) over time. Data outliers in 2007 for both of these parameters are likely due to a cohort effect (over-representation of acute seroconversions with shorter time between seroconversion and recruitment into the cohort). For HIV seroconverters classified as having had syphilis before HIV infection we found a statistically significant association with age and place of residence for the time period until 2004 - a finding reflecting the time-space evolution of the syphilis co-epidemic in MSM in Germany. This association with age and residence was not detected for HIV seroconverters classified as coincident syphilis/HIV infection. Otherwise, we could find no indication for any significant interaction of other identified time trends on the time trends for HIV-syphilis co-infection.

Syphilis circulation in the MSM population in Western Europe and North America reached a historic nadir during the 1990's. HSV-2, though declining in HIV-negative MSM as well, remained prevalent at high levels in HIV-positive men [9]. Epidemiological dynamics for these two sexually transmitted infections are quite different: syphilis usually becomes symptomatic, can be cured by antibiotics, and remains infectious only for a limited time period even without treatment. HSV-2 can only be suppressed - not cured - by antiviral drugs, and it establishes a persistent infection with recurrent viral shedding. Due to these differences, maintaining endemic levels of syphilis circulation requires a certain degree of delayed diagnosis, and core groups with high partner numbers - at least in countries with free access to healthcare, diagnosis and treatment.

The reasons for the re-emergence of syphilis in MSM populations in the last decade are not fully understood. Observations in Germany argue for the re-establishment of an endemic level of syphilis circulation in a population in which the infectious agent transiently almost had disappeared [11]. The transient disappearance may have been at least partly due to the shrinking of a core group that maintains uninterrupted syphilis circulation [12]. The reestablishment of this core group after 1996, rather than general increases in sexual risk 
behaviours of HIV-negative and untested MSM (e.g. increase of partner numbers and unprotected anal intercourse), may feed continuous syphilis circulation. Once the infectious agent - Treponema pallidum - became prevalent in these core groups again, it quickly reestablished a new level of endemicity (comparable to the pre-AIDS era). In the core groups, partner numbers and prevalence of unprotected anal intercourse are high - but unprotected sex takes place mostly within a context of HIV serosorting [13].

Syphilis and HIV surveillance data from Germany demonstrate transient increases in syphilis cases and newly diagnosed HIV in MSM, leveling off since 2004 (syphilis) and 2007 (HIV) (see supplemental online Figure 3). An increasing prevalence of syphilis in subpopulations at risk for HIV during this period could have resulted in an increase of HIV transmission probability due to effects of coincident syphilis on HIV susceptibility and infectivity. If our hypothesis is correct, the increase of HIV incidence in MSM should level off as soon as the new endemic level of syphilis circulation is reached - with a certain time delay due to a longer gap between infection and diagnosis for HIV compared with syphilis.

There are several limitations to our analysis: The analysis of the impact of other sexually transmitted infections on the risk of HIV seroconversion was not a primary aim of the HIV Seroconverter Cohort. Therefore the amount of data and information about the participants to investigate this issue is limited and in this respect the study suffers from the general drawbacks of a retrospective case-note review. The estimated dates of HIV infection in those cases in which no acute HIV infection was observed but the date was defined as the midpoint between the last negative and the first positive HIV antibody test [6], do not necessarily represent the actual date of infection.

Our group definitions based on antibody patterns are quite crude since there was no possibility to get information on the timing of suspicious clinical manifestations, and antibody 
titers at the time of syphilis diagnosis and treatment initiation were not available. Since the duration of infection and the baseline titers of syphilis antibodies at treatment initiation determine the time course of their following declines, there is certainly room for misclassifications. Misclassifications may occur in both directions: falsely classifying cases as coincident and falsely classifying cases as syphilis preceding HIV infection. Particularly in those patients in which the analyzed blood sample was collected more than 3 months after the estimated time of HIV infection, IgM and VDRL antibodies may have been eliminated already after adequate syphilis treatment, even if syphilis coincided with HIV transmission. Hence we feel that our classification system results in a rather conservative estimate of the proportion of coincident infections. Accordingly, group 1 with syphilis preceding HIV infection was the largest group.

A comparison of syphilis antibody prevalence in the group of HIV seroconverters presented here with a control group of HIV negative sexually active MSM from Berlin tested in 2006 showed a threefold higher syphilis seroprevalence in HIV seroconverters in 2006 (29\% vs. 9\%) [unpublished own data]. These data support the view, that coincident infections might be underestimated by the algorithm applied here.

Furthermore, the biological basis for the impact of syphilis on an individual's susceptibility for HIV infection has not yet been clearly defined. Besides direct interactions like breaks to the mucosal integrity, immunological interactions like increased concentration of potential HIV target cells at the portal of entry and increased CCR5 co-receptor expression may play a role. The evolution of these immunological factors over time and their dependence and interaction with syphilis treatment has not yet been elucidated in detail [14]. As demonstrated for HSV-2 infection, the period of hyper-susceptibility for HIV infection may extend beyond the active stage of disease [15]. 
One further aspect of syphilis as an HIV transmission co-factor which is not reflected in this analysis is an enhancing effect of syphilis co-infection in HIV infected individuals which results only in HIV transmission but not in syphilis transmission.

Participants of the HIV seroconverter study are primarily recruited in Germany's largest cities and thus do not represent HIV seroconverters in smaller cities and rural areas. Hence, our findings may not be generalizable to all MSM. However, German surveillance data show that HIV and syphilis diagnosis incidence increased coincidentally in all parts of Germany: the rise in syphilis incidence occurred first among gay communities in metropolitan areas, where a first peak occurred in 2003/2004, followed by transient declines (see surveillance data for Berlin, Figure 3). However, it could also be observed outside of the largest cities with a time delay of one to two years [2]. In non-metropolitan areas, the increase of newly diagnosed HIV-infections in parallel with increasing syphilis incidence was even more pronounced.

In summary, we found a considerable overlap of HSV-2 and syphilis infection and HIV acquisition among MSM/u in the German HIV Seroconverter Cohort. The time course and rate of co-infections suggest that the re-emergence of a syphilis co-epidemic among MSM after 2000 could have contributed to an increase of HIV incidence. Our data support the hypothesis that the increase of newly diagnosed HIV infections in MSM after the year 2000 may at least partly be a result of enhanced HIV transmission probability rather than general and widespread changes of HIV-related risk behaviors $[5 ; 16]$.

At the same time, the high prevalence of previous syphilis episodes before HIV seroconversion argues for targeted interventions to strengthen HIV protective behavior in HIV negative MSM who are diagnosed with newly acquired syphilis.

Experiences so far with addressing STI co-factors as a means to reduce HIV incidence have often been disappointing [17]. However, the high incidence and prevalence of syphilis in 
definable and accessible MSM subpopulations is by itself a reason for concern and should prompt increasing efforts to get this epidemic under control. Including syphilis into regular monitoring of HIV infected patients and improving sexual health care for at risk populations by low threshold sites for HIV/STI- diagnosis and treatment might be an approach that could be promoted and tested.

Word count: 3,029 


\section{Figures}

Figure 1: Flowchart of analysis and group assignments

Figure 2: Trends for syphilis co-infection in HIV seroconverters from Germany, 1996-2007

Proportions of HIV seroconverters with probable coincident syphilis, and syphilis infection preceding HIV infection, during the years 1996 to 2007 (with 95\%CI). The declining proportion of HIV seroconverters with coincident syphilis after 2003 reflects the course of the epidemic in Berlin (see Fig.3). The transient drop of coincident syphilis in 2004 may suggest a recruitment bias or group misclassification.

(supplemental) Figure 3: Newly diagnosed HIV in MSM, resp. syphilis in MSM and men with unknown transmission risk (MSM/u) in Germany resp. Berlin (B) from 2001 through 2008 (surveillance data*)

* due to a change in reporting procedures, syphilis data from before 2001 are not presented since they would not be not comparable to data collected from 2001 onwards 
Table 1: Time trends for syphilis co-infection and other potentially confounding parameters in the German HIV-1 Seroconverter Cohort

\begin{tabular}{|c|c|c|c|c|c|c|c|c|c|}
\hline & 1996-99 & 2000 & 2001 & 2002 & 2003 & 2004 & 2005 & 2006 & 2007 \\
\hline \# HIV seroconverters & 132 & 44 & 62 & 74 & 130 & 176 & 170 & 170 & 94 \\
\hline Mean age at seroconversion (in years) & 32.15 & 31.48 & 32.37 & 32.50 & 33.38 & 33.68 & 34.01 & 33.19 & 35.04 \\
\hline Proportion recruited in Berlin (\%) & 69.0 & 81.4 & 77.4 & 68.0 & 77.9 & 79.2 & 72.4 & 60.4 & 67.0 \\
\hline Ratio of documented/ acute seroconversion & 2.8 & 1.9 & 2.7 & 2.0 & 2.0 & 1.4 & 1.5 & 1.6 & 0.5 \\
\hline Mean time (months) from last negative to first positive HIV test $(n=969)$ & 11.95 & 12.14 & 13.63 & 13.93 & 14.13 & 15.97 & 14.49 & 14.46 & 14.68 \\
\hline Mean time (days) between calculated HIV seroconversion and blood & & & & & & & & & \\
\hline sample $(n=275)$ & 340 & 190 & 317 & 161 & 228 & 193 & 204 & 158 & 60 \\
\hline Proportion with syphilis antibodies (\%) & 9.8 & 13.6 & 21.0 & 23.0 & 33.1 & 30.7 & 34.7 & 28.2 & 23.4 \\
\hline Proportion with syphilis preceding HIV infection (\%) & 6.1 & 11.4 & 11.3 & 12.2 & 14.6 & 22.7 & 21.8 & 19.4 & 19.1 \\
\hline Proportion with syphilis coinciding with HIV infection (\%) & 2.3 & 2.3 & 6.5 & 8.1 & 16.9 & $8.0^{*}$ & 12.4 & 7.6 & 4.3 \\
\hline Proportion with HSV-2 antibodies (\%) & 44.7 & 31.8 & 33.3 & 42.7 & 42.0 & 39.8 & 39.4 & 42.9 & 38.3 \\
\hline Proportion with HSV-2 IgM (\%) & 7.0 & 7.1 & 12.7 & 12.3 & 10.1 & 10.1 & 12.3 & 12.8 & 16.1 \\
\hline Proportion with HSV-1 IgG antibodies (\%) & 86.9 & 95.5 & 95.2 & 91.5 & 89.1 & 90.1 & 90.4 & 88.8 & 92.5 \\
\hline
\end{tabular}

*The transient drop of coincident syphilis in 2004 may suggest a recruitment bias or group misclassification. 
Table 2: Results of Poisson regression for interaction of time dependent parameters (1996-2004) with proportion of HIV seroconverters with syphilis preceding HIV ( $\mathrm{n}=610)$

\begin{tabular}{|l|l|l|l|}
\hline & Prevalence ratio* & {$[95 \%$ conf. interval] } & P-value \\
\hline Date of infection (year) & 1.190 & $(1.066 ; 1.328)$ & 0.002 \\
\hline Berlin vs other regions & 1.849 & $(1.021 ; 3.348)$ & 0.043 \\
\hline Age at infection & 1.029 & $(1.004 ; 1.055)$ & 0.023 \\
\hline
\end{tabular}

*How to read prevalence ratios: syphilis antibody prevalence rate increased by $19.0 \%$ per year;

seroconverters from Berlin had a $84.9 \%$ higher prevalence compared to other seroconverters;

for each year of age the prevalence of a preceding syphilis infection increased by $2.9 \%$. 


\section{Acknowledgements:}

We wish to acknowledge the careful management of the stored plasma samples done by Sabrina Neumann and Hanno von Spreckelsen. Furthermore, we thank Axel J. Schmidt for critical review of the manuscript.

\section{Contributions of the authors:}

The study was conceived and supervised by U. Marcus. N. Spielmann analyzed the data and drafted the manuscript. D. Münstermann and H-J. Hagedorn supervised the antibody testing of the blood samples and participated in the development of the classification system for group assignment based on the antibody patterns. M. an der Heiden was responsible for the statistical and interaction analyses. C. Houareau extracted the relevant data from the HIV seroconverter cohort database. B. Gunsenheimer-Bartmeyer, C. Kücherer and K. Keeren coordinate and supervise the HIV seroconverter cohort and the sample collection and testing. O. Hamouda supervises the HIV seroconverter cohort and critically revised the manuscript. All authors contributed to the final manuscript.

\section{Competing interests:}

The authors declare that they have no competing interests.

\section{Funding:}

Funding for the German HIV Seroconverter Cohort was granted by the German Federal Ministry of Health.

\section{Copyright licence statement}

The Corresponding Author has the right to grant on behalf of all authors and does grant on behalf of all authors, an exclusive licence (or non-exclusive for government employees) on a worldwide basis to the BMJ Publishing Group Ltd and its Licensees to permit this article (if accepted) to be published in Sexually Transmitted Infections and any other BMJPGL products to exploit all subsidiary rights, as set out in our licence.

\section{Key Messages:}

- High proportions of MSM HIV seroconverters from the German HIV-1 seroconverter cohort had positive serological markers for syphilis and HSV-2.

- While the proportion of MSM seroconverters co-infected with HSV-2 remained fairly stable between 1996 and 2007, syphilis seromarkers increased significantly between 1999 and 2005.

- Further differentiation into syphilis infections which were probably coinciding with HIV infection shows a significant increase of co-infections between 1999 and 2003.

- The re-emergence of a syphilis co-epidemic among MSM in the last decade could have contributed to an increase in HIV incidence. 


\section{References:}

1 Sullivan PS, Hamouda O, Delpech V, et al. Reemergence of the HIV epidemic among men who have sex with men in North America, Western Europe, and Australia, 1996-2005. Annals of epidemiology 2009;19:423-431

2 Marcus U; Hamouda O. Syphilis in Germany, 2004: diagnoses increasing, particularly in smaller cities and rural areas. Euro Surveill 2005;10:E050728 050723

3 Marcus U, Schmidt AJ, Kollan C, and Hamouda O. The denominator problem: estimating MSM-specific incidence of sexually transmitted infections and prevalence of HIV using population sizes of MSM derived from Internet surveys. BMC public health 2009;9: 181

4 Marcus U, Schmidt AJ, Hamouda O, and Bochow M. Estimating the regional distribution of men who have sex with men (MSM) based on Internet surveys. BMC public health 2009; 9:180

5 Hoare A, Wilson DP, Regan DG, Kaldor J and Law MG. Using mathematical modelling to help explain the differential increase in HIV incidence in New South Wales, Victoria and Queensland: importance of other sexually transmissible infections. Sex Health 2008;5:169-187

6 Poggensee G, Kücherer C, Werning J, et al. Impact of transmission of drug-resistant HIV on the course of infection and the treatment success. Data from the German HIV-1 Seroconverter Study. HIV Medicine 2007;8:511-519

7 Jin F, Prestage GP, Mao L, et al. Transmission of herpes simplex virus types 1 and 2 in a prospective cohort of HIV-negative gay men: the health in men study. J Infect Dis. 2006;194:561-570

8 Frome EL. The analysis of rates using Poisson regression models. Biometrics 1983;39:665-674 
9 Smit C, Pfrommer C, Mindel A, et al. Rise in seroprevalence of herpes simplex virus type 1 among highly sexual active homosexual men and an increasing association between herpes simplex virus type 2 and HIV over time (1984-2003). Eur J Epidemiol 2007;22:937944

10 Hill C, McKinney E, Lowndes CM, et al. Epidemiology of herpes simplex virus types 2 and 1 amongst men who have sex with men attending sexual health clinics in England and Wales: implications for HIV prevention and management. Euro Surveill 2009;14(47):

11 Savage EJ, Hughes G, Ison C, et al. Syphilis and Gonorrhoea in men who have sex with men: A European overview. Euro Surveill 2009;14(47):pii=19417

12 Chesson HW, Gift TL. Decreases in AIDS Mortality and Increases in Primary and Secondary Syphilis in Men Who Have Sex With Men in the United States. J Acquir Immune Def Syndr 2008;47:263-264

13 Parsons JT, Schrimshaw EW, Wolitski RJ, et al. Sexual harm reduction practices of HIV-seropositive gay and bisexual men: serosorting, strategic positioning, and withdrawal before ejaculation. AIDS 2005;19:S13-S25

14 Salazar JC, Cruz AR, Pope CD, et al. Treponema pallidum elicits innate and adaptive cellular immune responses in skin and blood during secondary syphilis: a flow-cytometric analysis. Journal of Infectious Diseases 2007;195:879-887

15 Zhu J, Hladik F, Woodward A, et al. Persistence of HIV-1 receptor-positive cells after HSV-2 reactivation is a potential mechanism for increased HIV-1 acquisition. Nat Med 2009;15:886-892

16 Marcus U, Kollan C, Bremer V and Hamouda O. Relation between the HIV and the re-emerging syphilis epidemic among MSM in Germany: an analysis based on anonymous surveillance data. Sexually Transmitted Infections 2005;81:456-457

17 Celum C, Wald A, Lingappa JR, et al. Acyclovir and transmission of HIV-1 from persons infected with HIV-1 and HSV-2. N Eng J Med 2010; 10.1056/NEJMoa0904849 
Annex:

Participating Collaborators of the German HIV-1 Seroconverter Study Group

Aachen: Dres. Habets and Knechten; Augsburg: Dr. Hammond (Klinikum Augsburg);

Berlin: Dres. Mayr, Schmidt, Speidel, and Strohbach (Medizinisches Versorgungszentrum, Ärzteforum Seestraße), PD Dr. Arastéh (Auguste-Viktoria-Krankenhaus/Vivantes), Dres.

Bieniek and Cordes, Dr. Claus, Dres. Baumgarten, Carganico, and Dupke, Dres. Freiwald and Rausch, Dres. Gölz, Klausen, Moll, and Schleehauf, Dr. Hintsche, Dres. Jessen and Jessen, Dres. Köppe and Krauthausen, Dr. Reuter; Bielefeld: Dr. Pfaff (Krankenhaus MARA II); Bochum: Prof. Dr. Brockmeyer (St. Joseph-Hospital); Bonn: Prof. Dr. Rockstroh (Universitätsklinik Bonn); Dortmund: Prof. Dr. Gehring and Dr. Schmalöer and Dr. Hower (Klinikum Dortmund, ID27 Ambulanz); Dresden: Prof. Dr. Spornraft-Ragaller (Universitätsklinikum Dresden); Duisburg: Dr. Becker-Boost, Dr. Kwirant; Düsseldorf: Prof. Dr. Häussinger and PD Dr. Reuter (Universitätsklinik Düsseldorf); Frankfurt/Main: Prof. Dr. Helm (Universitätsklinik Johann-Wolfgang-Goethe-Universität); Frankfurt/Oder: Dr. Markus; Halle/Saale: Dr. Kreft (Universitätsklinik Martin-Luther-Universität); Hamburg: Prof. Dr. Plettenberg, Dr. Stoehr, Dr. Graefe, and Dr. Lorenzen (Institut für Infektionsmedizin, ifi, Allgemeines Krankenhaus St. Georg); Dres. Adam, Schewe, and Weitner, Dr. Fenske, Dr.Hansen, Prof. Dr. Stellbrink (Infektionsmedizinisches Zentrum Hamburg, ICH); Hannover: Prof. Dr. Schmidt (Medizinische Hochschule Hannover), Dres. Buck and Leugner; Koblenz: Prof. Dr. Eisenhauer and Dr. Rieke (Krankenhaus Kemperhof); Köln: Dr. Bihari, Dr. Ferdinand, Prof. Dr. Fätkenheuer (Universitätsklinik Köln); Prof. Dr. Oette, Krankenhaus der Augustinerinnen, Köln; Leipzig: Dr. Pfeil (Universitätsklinik Leipzig); Magdeburg: Prof. Dr. Malfertheiner and Dr. Wolle (Universitätsklinik Otto-v.-Guericke-Universität); Mainz: Prof. Dr. Galle (Klinikum der Joh.-Gutenberg-Universität); München: Dres. Jäger and Jägel-Guedes, Dr. Malm, Dr. Rieger, Städtisches Krankenhaus 12 München-Schwabing, Prof. Dr. Fröschl (Technische Universität München); Münster: Prof. Dr. Rahn, (Universitätsklinik Münster); Norderstedt: Dr. Soldan; Nürnberg: Dr. Brockhaus (Klinikum Nürnberg); Osnabrück: Dr. Mutz (Städtische Klinik Natruper Holz); Regensburg: Prof. Dr. Salzberger, Prof. Dr. Schölmerich, and Dr. Schneidewind (Universitätsklinik Regensburg); Remscheid: Dr. Steege; Rostock: Dr. Kreft, Prof. Dr. Ziegler and Prof. Dr. Reisinger (Universitätsklinik Rostock); Stuttgart: Dres. Ißler, Schaffert, Schnaitmann, and Trein, Dres. Frietsch, Müller, and Ulmer; Dr. Wagner-Wiening (Landesgesundheitsamt Stuttgart); Ulm: Prof. Dr. Kern and Prof. Dr. Dr. Kreidler (Universitätsklinik Ulm); Viernheim: Dr. van Treek; Wiesbaden: Dr. Starke 


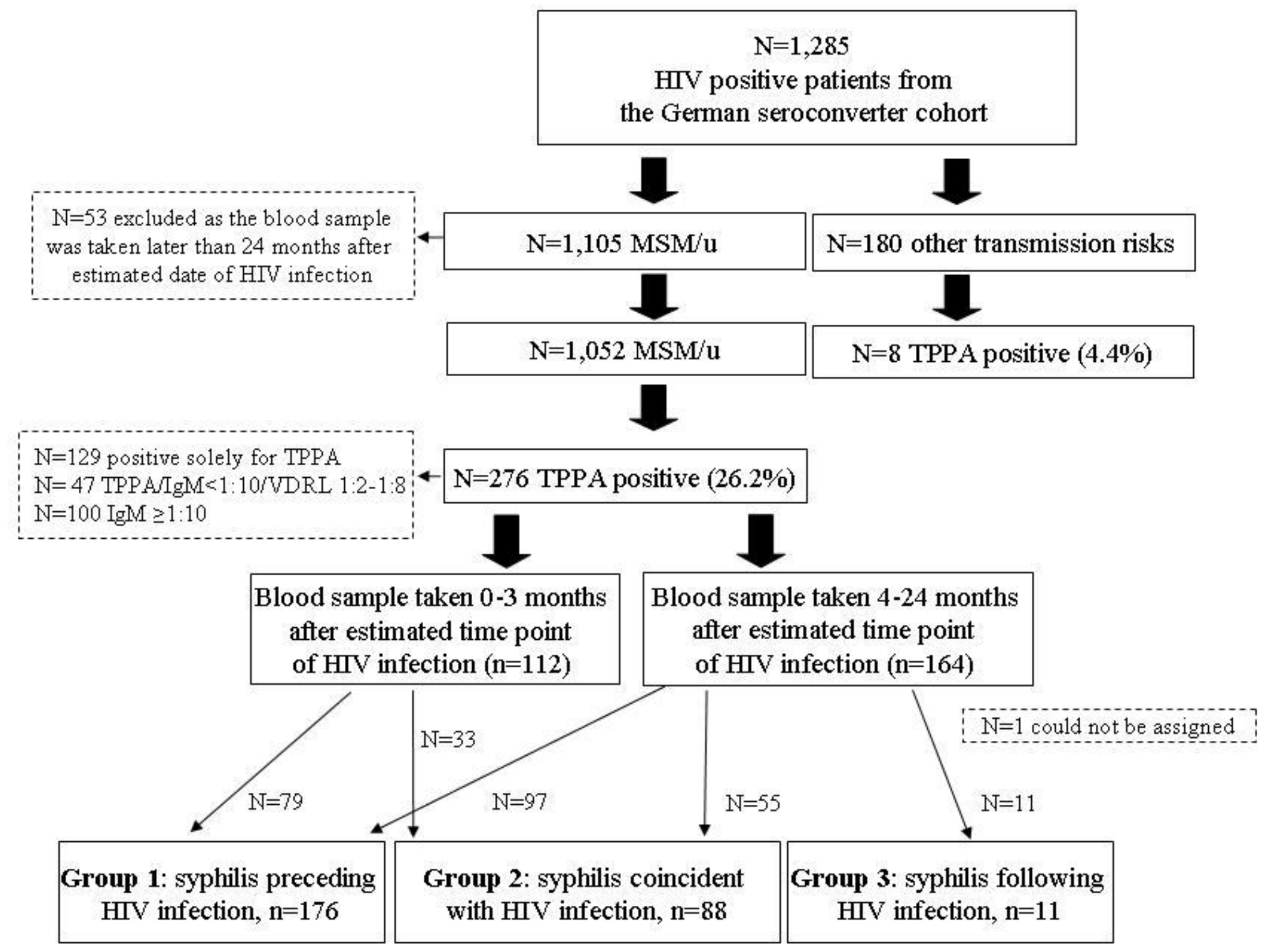




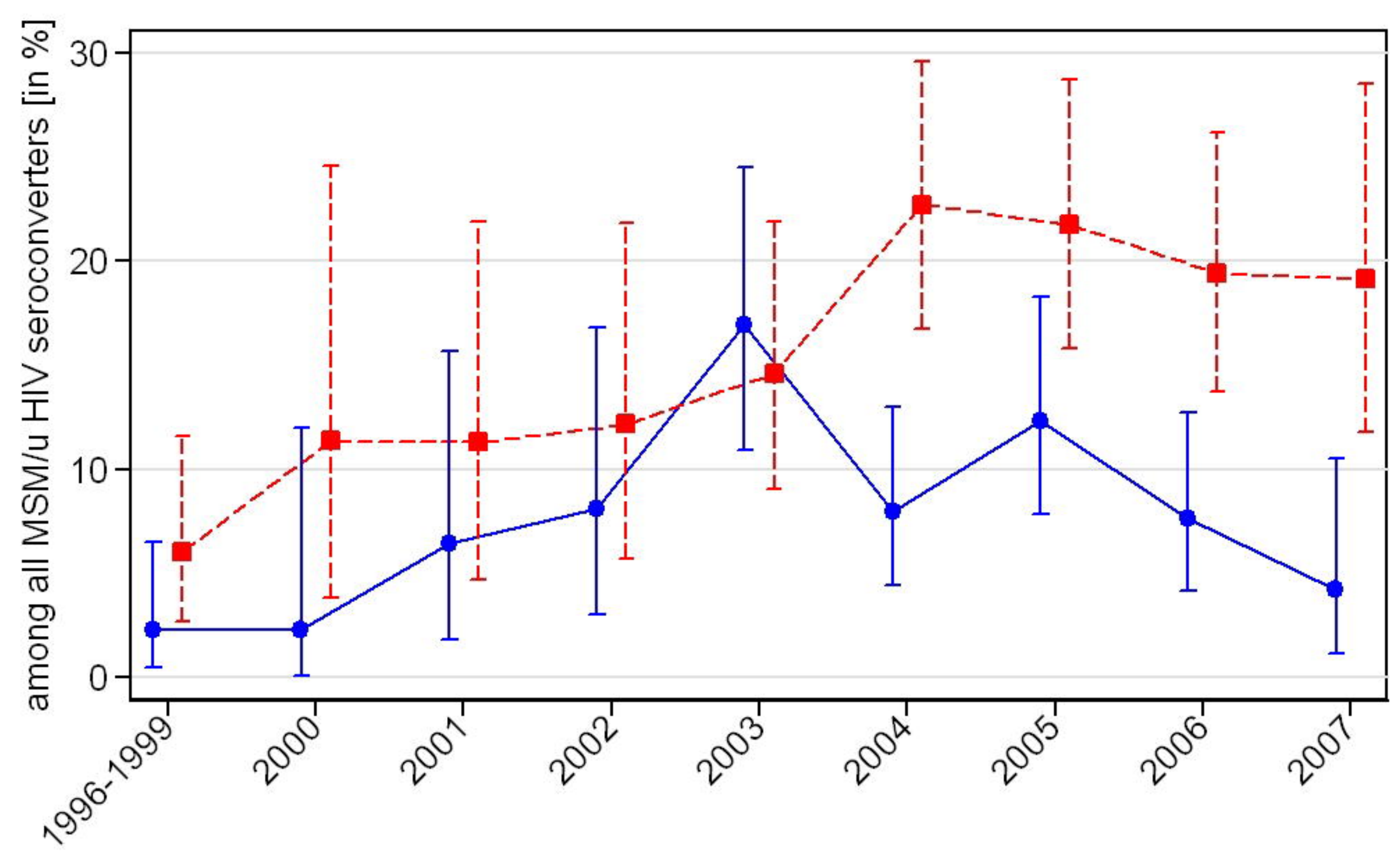

year of HIV infection

Syphilis $\longrightarrow$ coincident with $\quad---\cdots$ precedes HIV infection 